\title{
Production cross sections of cosmic antiprotons in the light of new data from the NA61 and LHCb experiments
}

\author{
Fiorenza Donato* \\ Dipartimento di Fisica, Università di Torino, Via P. Giuria 1, 10125 Torino, Italy \\ Istituto Nazionale di Fisica Nucleare, Sezione di Torino, Via P. Giuria 1, 10125 Torino, Italy \\ E-mail: donato@to.infn.it

\section{Michael Korsmeier} \\ Dipartimento di Fisica, Università di Torino, Via P. Giuria 1, 10125 Torino, Italy \\ Istituto Nazionale di Fisica Nucleare, Sezione di Torino, Via P. Giuria 1, 10125 Torino, Italy \\ Institute for Theoretical Particle Physics and Cosmology, RWTH Aachen University, \\ Sommerfeldstr. 16, 52056 Aachen, Germany E-mail: mi chael . korsmeier@to. infn. it

\section{Mattia di Mauro} \\ NASA Goddard Space Flight Center, Greenbelt, MD 20771, USA \\ Catholic University of America, Department of Physics, Washington DC 20064, USA
}

\begin{abstract}
The cosmic-ray flux of antiprotons is measured with unprecedented precision by the particle spectrometer AMS-02 on the International Space Station. Its interpretation requires a correct description of the dominant production process for antiprotons in our Galaxy, namely, the interaction of cosmic-ray proton and helium with the atoms of the interstellar medium. In light of new cross section measurements by the NA61 experiment of $p+p \rightarrow \bar{p}+X$ and the first ever measurement of $p+\mathrm{He} \rightarrow \bar{p}+X$ by the LHCb experiment in a fixed target configuration, we update the parametrization of proton-proton and proton-nucleon cross sections and their uncertainties. The new parametrizations for the cross section are used to derive the total source term of secondary antiprotons. Since uncertainties, in particular at low energies, are significantly larger than those on the measured antiproton flux by the AMS-02 experiment, we finally quantify the necessity of new data on antiproton production cross sections, and pin down the kinematic parameter space which should be covered by future high-energy experiments.
\end{abstract}

36th International Cosmic Ray Conference -ICRC2019-

July 24th - August 1st, 2019

Madison, WI, U.S.A.

${ }^{*}$ Speaker. 


\section{Introduction}

With the last generation of particle detectors in space, physics of charged cosmic rays (CRs) has become a precision discipline. During the last decade, the space-based spectrometers PAMELA and AMS-02, which is borne to the International Space Station, have driven measurement uncertainties in the CR fluxes as low as the percent level in an energy range from $1 \mathrm{GeV}$ to a few TeV. They have measured the CR nuclear [1, 2, 3, 4, 5] and leptonic (positron and electron) $[6,7,8,9,10]$ components, as well as CR antiprotons [11, 12]. The most recent antiproton flux measurement by AMS-02 extends from 1 to $400 \mathrm{GeV}$ with an uncertainty of 5\% for almost the whole energy range. It is generally established that the bulk of antiprotons in our Galaxy is produced by the interaction of CRs on the interstellar medium (ISM) [13], conventionally called secondary antiprotons. The dominant contribution is provided by the proton-proton $(p p)$ channel, namely CR proton on ISM hydrogen, and either the CR projectile or the ISM target replaced by helium (He $p, p \mathrm{He}$, and $\mathrm{HeHe}$ ).

In reference [14] (hereafter DKD17), we discussed the requirement on cross section measurements to determine the antiproton source term at the uncertainty level of AMS-02 flux data. In [15] (hereafter KDD18) we seek to determine the source term and its uncertainty from existing cross section measurements.

\section{The antiproton source term}

The source term of antiproton $q_{i j}$ is given by the convolution of the CR flux $\phi_{i}$, the ISM density $n_{\mathrm{ISM}, j}$, and the production cross section $\sigma_{i j}$ as stated in

$$
q_{i j}\left(T_{\bar{p}}\right)=\int_{T_{\mathrm{th}}}^{\infty} d T_{i} 4 \pi n_{\mathrm{ISM}, j} \phi_{i}\left(T_{i}\right) \frac{d \sigma_{i j}}{d T_{\bar{p}}}\left(T_{i}, T_{\bar{p}}\right) .
$$

Here $T_{i}$ is the CR projectile energy and $T_{\bar{p}}$ the kinetic energy of the produced antiproton. The dominant channels for the production of secondary antiprotons are $p p$ at roughly 50-60\% of the total spectrum, and $p \mathrm{He}$ and $\mathrm{He} p$ at 10-20\% each, while the channels involving heavier incoming CRs contribute only up to a few percent (see below). Until very recently, no measurements of the helium channels were available, rendering the $p p$ channel the baseline for any scaling to protonnucleus $(p A)$ or nucleus-nucleus $(A A)$ channels.

Figure 1 shows this relative contribution to the source term for different high-energy experiments in the proton-proton and proton-nucleus production channels. We note in particular two points: Firstly, in the $p p$ channel recent experimental data by NA49 [16] and NA61 [17] only cover the source term well above $T_{\bar{p}} \sim 5 \mathrm{GeV}$. Secondly, due to the large projectile proton energy the LHCb data [18] in the $p \mathrm{He}$ channel contribute to the source term only at very high energies and at a low ratio.

\section{Methods}

High-energy experiments measure the fully-differential cross section of antiproton production as function of center-of-mass energy, $\sqrt{s}$, antiproton transverse momentum, $p_{\mathrm{T}}$, and Feynman 
scaling $x_{f}$, conventionally expressed in the Lorentz-invariant form

$$
E \frac{d^{3} \sigma_{p+p \rightarrow \bar{p}}}{d p^{3}}\left(\sqrt{s}, p_{\mathrm{T}}, x_{f}\right)
$$

We fit the two most recent analytic parametrization suggested by Di Mauro et al. (parametrization I) [19] and Winkler (parametrization II) [20], respectively, to new data provided by NA61 and LHCb as summarized in Table 1. To obtain the energy-differential cross section which enters the source term integral, Eq. (1), we integrate over all angles. Figure 2 displays this energy-differential
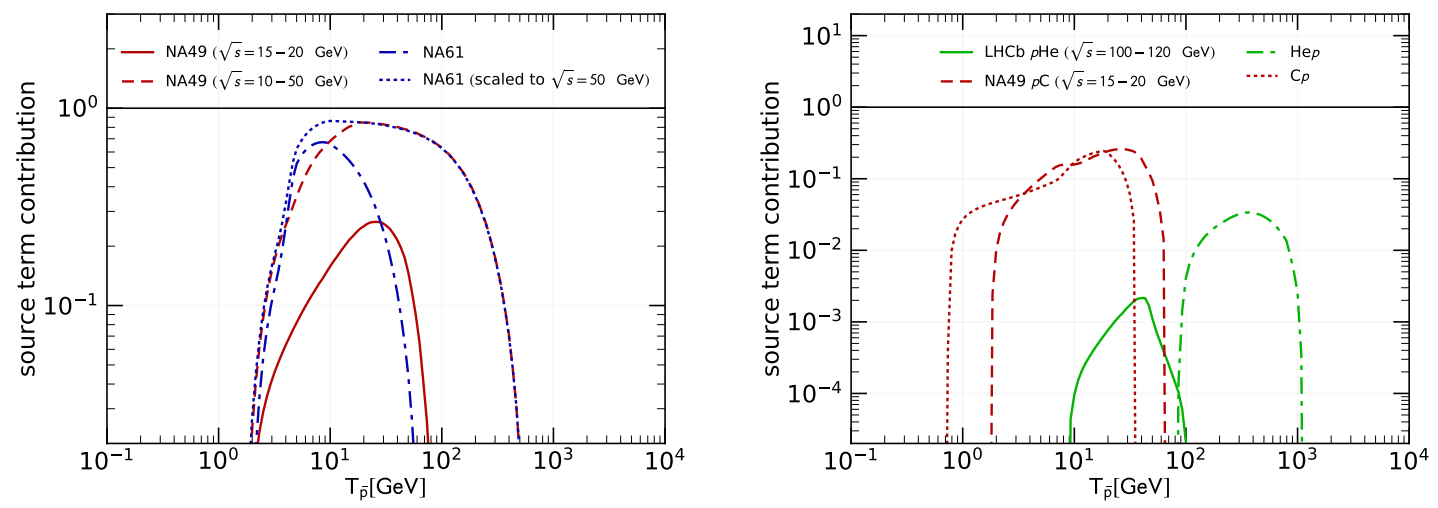

Figure 1: Left: Fraction of the $p p$ source term originating from the kinematic parameter space of the cross section which is experimentally determined by NA49 and NA61. The contribution is normalized to the total $p p$ source term. The NA61 data are taken for $\sqrt{s}=7.7 \mathrm{GeV}$ to $17.3 \mathrm{GeV}$ (blue dot-dashed line), while the NA49 is taken at $\sqrt{s}=17.3 \mathrm{GeV}$ and here assumed to be valid in the range $15-20 \mathrm{GeV}$ (solid red line). The red dashed line is obtained assuming that the NA49 data are valid in the $\sqrt{s}$ range from 10 to $50 \mathrm{GeV}$, while the dotted blue one is obtained extending the validity of NA61 data up to $\sqrt{s}=50 \mathrm{GeV}$. Right: as in the left panel, but for the kinematic parameter space of the cross section which is experimentally determined by $\mathrm{NA} 49 p \mathrm{C}$ and $\mathrm{LHCb} p \mathrm{He}$ data. Both panels are taken from KDD18.

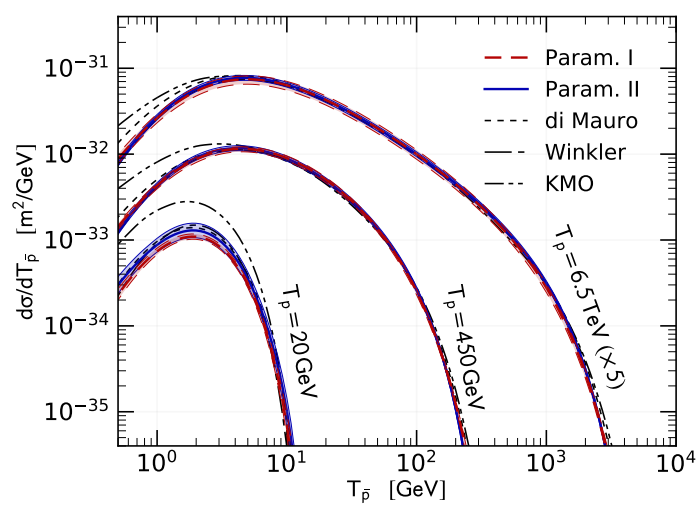

Figure 2: The differential cross section $d \sigma / d T_{\bar{p}}(p+p \rightarrow \bar{p}+X)$ for prompt antiprotons, at the representative proton energies $T_{p}=20 \mathrm{GeV}, 450 \mathrm{GeV}$ and $6.5 \mathrm{TeV}$. The dashed (solid) line and the red (blue) band are the result of our analysis for Param. I and Param. II. We report for comparison some literature estimations (see text for details). Tables with the full cross section results are provided in the supplementary material to this paper. Figure is taken from KDD18. 


\begin{tabular}{lc}
\hline \hline Experiment & $\sqrt{s}[\mathrm{GeV}]$ \\
\hline NA49 & 17.3 \\
NA61 & $\mathbf{7 . 7 , 8 . 8 , 1 2 . 3 , \mathbf { 1 7 . 3 }}$ \\
Dekkers et al. & $6.1,6.7$ \\
BRAHMS & 200 \\
\hline NA49 $(p$ C $)$ & 17.3 \\
LHCb $(p \mathbf{H e})$ & $\mathbf{1 1 0}$ \\
\hline \hline
\end{tabular}

Table 1: List and available CM energies of the data sets employed in our analysis.

cross section as function of the antiproton kinetic energy, $T_{\bar{p}}$, for three fixed proton energies, $T_{p}$. Both energies are given in the ISM rest frame. The two fitted parametrizations agree well within their uncertainties and reasonably within the original parametrization, labelled Winkler and $D i$ Mauro. However, the Monte Carlo driven approach by [21] shows significant deviations at low energies. Finally we note, that $\mathrm{LHCb}$ data at high energies give a preference for parametrization II. For details we refer to KDD18.

\section{Results}

We use the updated parametrization II to calculate the total source term of CR antiprotons, which includes also the contribution from antineutrons and anithyperons subsequently decaying into antiprotons. The results are shown in Fig. 3. The dominant contribution arise from the $p p$

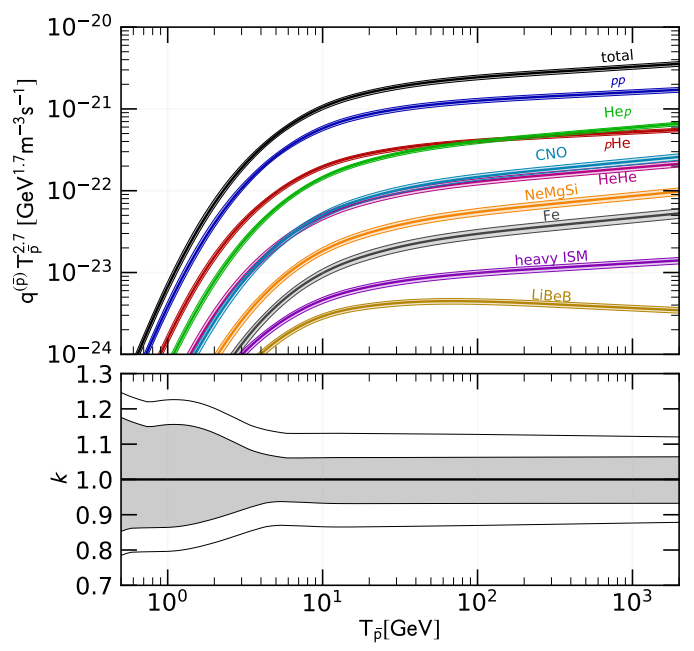

Figure 3: Source terms of CR antiprotons and separate CR-ISM contributions. The shaded bands report the $2 \sigma$ uncertainty due to prompt $\bar{p}$ production cross sections. In the bottom panel we show the relative uncertainty on the total source term. The grey band refers to the prompt $\bar{p}$ 's only, while the outer lines quantify the additional uncertainty due to isospin violation and to hyperons decay. The figure is taken from KDD18. 
channel, $50 \%$ to $60 \%$, and the $\mathrm{He} p$ and $p \mathrm{He}$ channels with $15 \%$ to $20 \%$ each. At intermediate energies from $T_{\bar{p}}=5 \mathrm{GeV}$ up to a few hundred $\mathrm{GeV}$ the prompt source terms are affected by an uncertainties of $\pm 8 \%$ at the $2 \sigma$ level and increase to $\pm 15 \%$ below $T_{\bar{p}}=5 \mathrm{GeV}$. Antineutron- and hyperon-induced production increases the uncertainty by an additional $5 \%$.

\section{Requirements for future experiments}

In order to improve the current situation where uncertainties in the prediction of the antiproton source term significantly overshoot the uncertainty of the antiproton flux measurement by the AMS-02 experiment. We propose new cross section measurement and in Fig. 4 quantify the kinetic parameter space which is relevant for CR physics. If cross sections where measured with $3 \%$ uncertainty inside the blue contours and known with $30 \%$ uncertainty everywhere else we could predict the antiproton flux at same accuracy as measured by AMS-02. We note that new measurements would be in particular important at low energies where the shape of the cross section is more uncertain.

\section{Conclusions}

The role of high-energy particle physics in the interpretation of CR data receives increasing attention, since data from space are provided with improving precision. In this contribution, we present the analysis of the first-ever data on the inclusive cross section $p+\mathrm{He} \rightarrow \bar{p}+X$ collected by the LHCb collaboration at Cern, with beam protons at $T_{p}=6.5 \mathrm{TeV}$ and a fixed helium target. We use our updated cross sections to compute the antiproton source terms and their uncertainties for all the production channels, considering also nuclei heavier than He both in CRs and the ISM. Finally, we quantify the necessity of new data on antiproton production cross sections, and pin
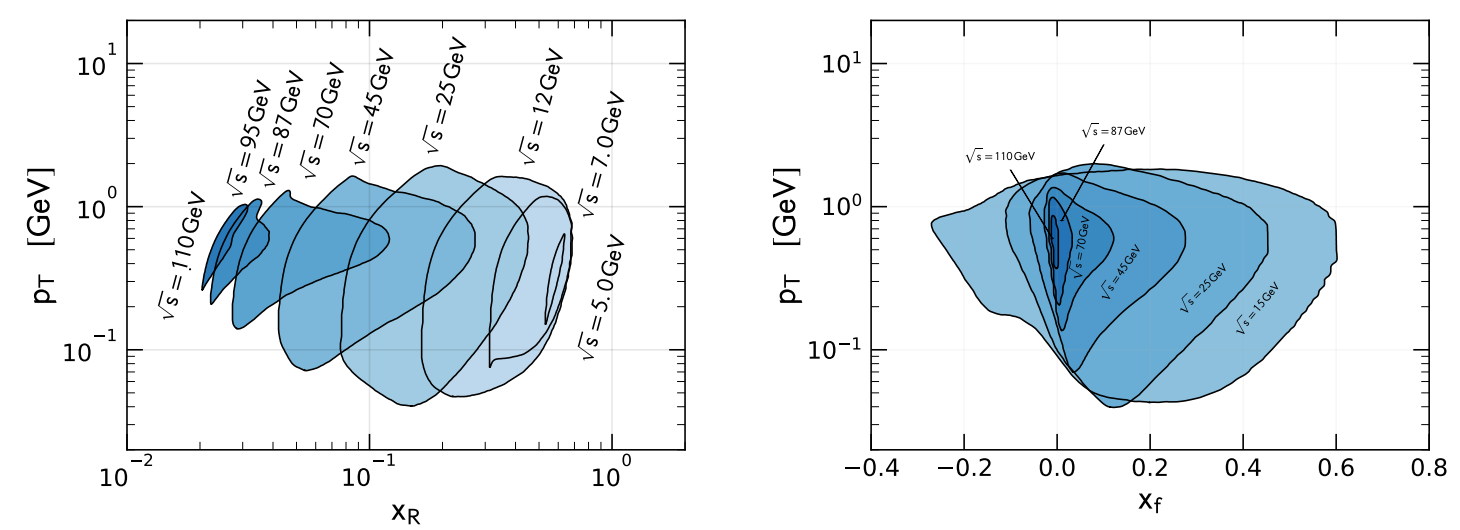

Figure 4: Parameter space of the antiproton production cross section which is necessary to determine the antiproton source term at the uncertainty level of AMS-02 measurements [12]. We require the cross section to be known by $3 \%$ within the blue shaded regions and by $30 \%$ outside of the contours. The left panel (taken from DKD17) shows the kinetic parameter space for different $\sqrt{s}$ as function of radial scaling $x_{R}$ and transverse momentum $p_{T}$, while in the right panel (taken from KDD18) we replace the radial scaling by the Feynman scaling $x_{f}$. Note that the kinetic parameter space shown in the left and right panel is identical. 
down the kinematic parameter space which should be covered by future data. For further details on the topics treated in the present paper, we refer to DKD17 and KDD18.

\section{References}

[1] PAMELA collaboration, PAMELA Measurements of Cosmic-ray Proton and Helium Spectra, Science 332 (2011) 69 [1103.4055].

[2] O. Adriani et al., Measurement of Boron and Carbon Fluxes in Cosmic Rays with the PAMELA Experiment, Astrophys. J. 791 (2014) 93 [1407.1657].

[3] AMS collaboration, Precision Measurement of the Proton Flux in Primary Cosmic Rays from Rigidity 1 GV to 1.8 TV with the Alpha Magnetic Spectrometer on the International Space Station, Phys. Rev. Lett. 114 (2015) 171103.

[4] AMS collaboration, Precision Measurement of the Helium Flux in Primary Cosmic Rays of Rigidities 1.9 GV to 3 TV with the Alpha Magnetic Spectrometer on the International Space Station, Phys. Rev. Lett. 115 (2015) 211101.

[5] AMS collaboration, Precision Measurement of the Boron to Carbon Flux Ratio in Cosmic Rays from 1.9 GV to 2.6 TV with the Alpha Magnetic Spectrometer on the International Space Station, Phys. Rev. Lett. 117 (2016) 231102.

[6] O. Adriani et al., An anomalous positron abundance in cosmic rays with energies $1.5-100 \mathrm{GeV}$, nature 458 (2009) 607 [0810 . 4995].

[7] O. Adriani et al., Cosmic-Ray Electron Flux Measured by the PAMELA Experiment between 1 and 625 GeV, Phys. Rev. Lett. 106 (2011) 201101 [1103.2880].

[8] M. Aguilar, G. Alberti, B. Alpat, A. Alvino, G. Ambrosi, K. Andeen et al., First Result from the Alpha Magnetic Spectrometer on the International Space Station: Precision Measurement of the Positron Fraction in Primary Cosmic Rays of 0.5-350 GeV, Phys. Rev. Lett. 110 (2013) 141102.

[9] M. Aguilar, D. Aisa, A. Alvino, G. Ambrosi, K. Andeen, L. Arruda et al., Electron and Positron Fluxes in Primary Cosmic Rays Measured with the Alpha Magnetic Spectrometer on the International Space Station, Phys. Rev. Lett. 113 (2014) 121102.

[10] M. Aguilar, D. Aisa, B. Alpat, A. Alvino, G. Ambrosi, K. Andeen et al., Precision Measurement of the $\left(e^{+}+e^{-}\right)$Flux in Primary Cosmic Rays from $0.5 \mathrm{GeV}$ to $1 \mathrm{TeV}$ with the Alpha Magnetic Spectrometer on the International Space Station, Phys. Rev. Lett. 113 (2014) 221102.

[11] O. Adriani et al., PAMELA Results on the Cosmic-Ray Antiproton Flux from $60 \mathrm{MeV}$ to $180 \mathrm{GeV}$ in Kinetic Energy, Phys. Rev. Lett. 105 (2010) 121101 [1007.0821].

[12] AMS collaboration, Antiproton Flux, Antiproton-to-Proton Flux Ratio, and Properties of Elementary Particle Fluxes in Primary Cosmic Rays Measured with the Alpha Magnetic Spectrometer on the International Space Station, Phys. Rev. Lett. 117 (2016) 091103.

[13] G. Giesen, M. Boudaud, Y. Genolini, V. Poulin, M. Cirelli, P. Salati et al., AMS-02 antiprotons, at last! Secondary astrophysical component and immediate implications for Dark Matter, JCAP 1509 (2015) 023 [1504.04276].

[14] F. Donato, M. Korsmeier and M. Di Mauro, Prescriptions on antiproton cross section data for precise theoretical antiproton flux predictions, Phys. Rev. D96 (2017) 043007 [170 4 . 03663]. 
[15] M. Korsmeier, F. Donato and M. Di Mauro, Production cross sections of cosmic antiprotons in the light of new data from the NA61 and LHCb experiments, Phys. Rev. D97 (2018) 103019 [1802.03030].

[16] NA49 collaboration, Inclusive production of protons, anti-protons and neutrons in $p+p$ collisions at 158-GeV/c beam momentum, Eur. Phys. J. C65 (2010) 9 [0904.2708].

[17] NA61/SHINE collaboration, Measurements of $\pi^{ \pm}, K^{ \pm}, p$ and $\bar{p}$ spectra in proton-proton interactions at 20,31, 40,80 and $158 \mathrm{GeV} / \mathrm{c}$ with the NA61/SHINE spectrometer at the CERN SPS, Eur. Phys. J. C77 (2017) 671 [1705.02467].

[18] LHCB collaboration, Measurement of antiproton production in pHe collisions at sqrts $=110 \mathrm{GeV}$, in 52nd Rencontres de Moriond, 2017, https://cds.cern.ch/record/2260835/files/LHCb-CONF-2017-002.pdf.

[19] M. di Mauro, F. Donato, A. Goudelis and P. D. Serpico, New evaluation of the antiproton production cross section for cosmic ray studies, Phys. Rev. D90 (2014) 085017 [1408 . 0288].

[20] M. W. Winkler, Cosmic Ray Antiprotons at High Energies, JCAP 1702 (2017) 048 [1701. 04866 ].

[21] M. Kachelriess, I. V. Moskalenko and S. S. Ostapchenko, New calculation of antiproton production by cosmic ray protons and nuclei, Astrophys. J. 803 (2015) 54 [1502 . 04158 ]. 